\title{
Glutathione-Responsive Carboxymethyl Chitosan Nanoparticles for Controlled Release of Herbicides
}

\author{
Zhiyan Yu*, Xin Sun, Haixia Song, Wenqian Wang, Zhao Ye, Liyan Shi, Kaikai Ding \\ Department of Pharmaceutical Engineering, School of Chemistry, Chemical Engineering and Life Sciences, \\ Wuhan University of Technology, Wuhan, China \\ Email: 991174617@qq.com
}

Received 15 May 2015; accepted 26 June 2015; published 29 June 2015

Copyright (C) 2015 by authors and Scientific Research Publishing Inc.

This work is licensed under the Creative Commons Attribution International License (CC BY). http://creativecommons.org/licenses/by/4.0/

C) (7) Open Access

\begin{abstract}
Glutathione-responsive carboxymethyl chitosan nanoparticles cross-linked with disulfide bonds were developed for controlled release of herbicides. The nanoparticles were synthesized by selfassembly of amphiphilic carboxymethyl chitosan derivative (CMCS-MUA) in aqueous solution and subsequently producing disulfide cross-linking bonds by ultrasonic treatment. TEM showed that the nanoparticles had a spherical core-shell configuration with a size of about $250 \mathrm{~nm}$. Assessment of stability of the nanoparticles (considering mean diameter, polydispersity, and Zeta potential) was conducted over a period of three months, and the nanoparticles were found to be stable in solution. Herbicide-loaded nanoparticles were prepared using diuron as a model herbicide. In vitro release study revealed that diuron can be released from nanoparticles in a controlled manner depended on the glutathione concentration. Herbicidal activity assays performed with preemergence treatment of target species (Echinochloa crusgalli) showed the effectiveness of diuron-loaded nanoparticles. Assays with nontarget species (Zea mays) showed that the diuronloaded nanoparticles did not affect plant growth. The results indicate that the glutathioneresponsive nanoparticles prepared in this work will be a promising candidate for controlled release of herbicides in agriculture.
\end{abstract}

\section{Keywords}

Glutathione-Responsive, Nanoparticles, Controlled Release, Herbicides, Diuron

\footnotetext{
${ }^{*}$ Corresponding author.
}

How to cite this paper: Yu, Z.Y., Sun, X., Song, H.X., Wang, W.Q., Ye, Z., Shi, L.Y. and Ding, K.K. (2015) Glutathione-Responsive Carboxymethyl Chitosan Nanoparticles for Controlled Release of Herbicides. Materials Sciences and Applications, 6, 591604. http://dx.doi.org/10.4236/msa.2015.66062 


\section{Introduction}

In recent years, with the rapid economic development, more attention have been paid to environmental issues caused by large-scale use of herbicides, which have become one of the most serious pollutants in surface and subterranean hydrological system worldwide. Reducing the use of herbicides and avoiding its undesirable impacts are considered as a part of the drive toward sustainable agriculture. Therefore, tremendous efforts have been devoted to the development of controlled release system for herbicides [1]-[5]. Controlled release is the permeation-regulated transfer of an ingredient from a reservoir to a targeted surface to maintain a predetermined concentration level for a specified period of time [6]. Compared to conventional herbicide formulations, controlled release herbicides have some advantages as follows: 1) longer residual biological activity; 2) controlling or reducing evaporation of herbicides; 3) increasing safety to non-target organisms including humans; 4) reducing the amount of herbicides applied; 5) preventing herbicides from escaping into the environment [7]. An ideal controlled release system for herbicides should not only efficiently load herbicides, protect herbicides before getting to the target sites, release the loaded herbicides at the target sites, but also maintain herbicides level adequate for weeds control for a period of time. Encapsulation of herbicides in a polymeric matrix can help to achieve these goals.

Recently with the development of nanotechnology, polymeric nanocarriers, which can transport active compounds like small molecular drugs, peptides, or genes, have get more and more attention. Especially in the drug delivery system, polymeric nanocarriers can enhance the solubility, bioavailability of the drug, and prolonged its circulation times, and most importantly, they can be tailor-made in such a manner that they selectively release their cargo at the desired site of action. So far, many smart drug delivery systems have been designed in response to certain stimuli, such as $\mathrm{pH}$, temperature, redox potential, enzymes, light, and so on.

Among these smart drug delivery systems, glutathione-responsive nanocarriers containing disulfide bonds have received considerable attention. They can be designed for intracellular drug delivery by taking advantage of the glutathione differences between extracellular and intracellular environment [8]. Glutathione (GSH) is an important natural tripeptide ( $\gamma$-L-glutamyl-L-cysteinyl -L-glycine) extensively existing in humans. In view of reports in the literature, disulfide bonds can keep stable in extracellular fluids (2 - $20 \mu \mathrm{M} \mathrm{GSH})$, whereas can be cleaved in an intracellular reductive environment (2 - $10 \mathrm{mM} \mathrm{GSH}$ ) [9]. Furthermore, the GSH concentration in some tumor cells (e.g. human lung adenocarcinoma A549 cells) has been reported to be several times higher than that in normal cells [10]. Most researches have been focused on introducing disulfide bonds into nanocarriers for controlled release of drugs in tumor cells [11]-[14]. However, up to now, there have been no studies about glutathione-responsive nanocarriers for herbicides applied in plants.

It is very interesting to note that GSH is widely existed not only in human cells but also in plant cells. According to the literature [15], GSH is present in various plant tissues at concentration of $2 \mathrm{mM}$ to $3 \mathrm{mM}$, in which disulfide bonds can be cleaved. Based on these principles, we constructed such a kind of glutathioneresponsive nanoparticle system for controlled release of herbicides. Chitosan was chosen as matrix for this system due to its nontoxicity and biodegradability, and disulfide bonds were introduced as the drug release switcher. Herbicides can be encapsulated into this nanosystem that is cross-linked by disulfide bonds. It is crucial that the encapsulated herbicide is well protected and will not leak out before it gets to the target plant. Once nanocarrier is exposed to the reductive glutathione environment after entering the plant cells, the disulfide bonds are cleaved, and therefore herbicide loaded in the nanocarrier is released. This glutathione-responsive nanoparticle system containing disulfide bonds designed in this paper may hold vast potential for efficient controlled release of herbicides in plants.

The objective of this work was to prepare such a glutathione-responsive system for controlled release of herbicides. The glutathione-responsive system constructed in this paper could not only uesd as a new type of carrier for controlled release of herbicides, avoding some disadvantage produced by the conventional herbicide formulations, but also could response to the GSH in plant cells so as to release the herbicide encapsulated in an intelligent responsive manner, which would have good application prospect in drug delivery field.

In this paper, first, an amphiphilic carboxymethyl chitosan derivative (CMCS-MUA) was synthesized through the chemical modification of chitosan using chloroacetic acid and 11-Mercapto undecanoic acid (MUA). Second, the CMCS-MUA was self-assembled into core-shell nanoparticles in aqueous solution driven by hydrophobic interaction. Then the disulfide cross-linked core-shell nanoparticles were obtained by ultrasonic treatment. The morphology, size distribution and Zeta potential of the nanoparticles were characterized by transmission electron microscopy (TEM) and dynamic light scattering (DLS). The pH sensitivity and the GSH responsiveness 
were also investigated. At last, diuron, a hydrophobic compound, was chosen as a model herbicide. By taking advantage of self-assemble behavior, diuron was encapsulated into the disulfide cross-linked nanoparticles. The drug release behavior of nanoparticles was studied by imitating the intracellular environment of plants cells, and the herbicidal activity was evaluated by means of pre-emergence treatment.

\section{Materials and Methods}

\subsection{Materials}

Chitosan (CS, MW = 560,000 Da, deacetylation degree = 91.13\%) was supplied from the Zhejiang Yuhuan Biotechnology Company. Chloroacetic acid (99.5\% purity) was obtained from Tianjin Fuchen Chemical Reagents Factory. 11-Mercapto undecanoic acid (MUA, 95.0\% purity) was purchased from Aldrich-Sigma. 1-(3Dimethylaminopropyl)-3-ethylcarbodiimide hydrochloride (EDC·HCl, 98.5\% purity), N-Hydroxy- succinimide (NHS, 98\% purity) were obtained from Aladdin. Diuron was provided by Wuhan Huancheng Science and Technology Ltd. All other chemical reagents in the study were of analytical grade.

\subsection{Synthesis of Carboxymethyl Chitosan (CMCS)}

According to the literature reported [16]-[20], carboxymethyl chitosan (CMCS) can be successfully prepared though following steps. Briefly, $8 \mathrm{~g}$ of chitosan was suspended in isopropanol (100 $\mathrm{mL})$ at room temperature while being stirred for $6 \mathrm{~h}$ and then filtered. The filter residue was treated with $\mathrm{NaOH}$ solution (50\%, wt\%), and this solution was fully stirred to mix evenly and then put in the refrigerator, where it froze overnight. Chloroacetic acid $(22 \mathrm{~g})$ dissolved in isopropanol $(28 \mathrm{~mL})$ was then dropped onto the frozen chitosan mixture, and the mixture was maintained under agitation for $20 \mathrm{~h}$ at a low temperature below $10^{\circ} \mathrm{C}$. Then the solution was filtered and the filter residue was washed with absolute ethanol. The crude product was purified and finally dried under vacuum. The N, O-substitution degree of carboxymethyl groups determined by elemental analysis was about 0.68 , and the degree of $\mathrm{N}$-substitution was about 0.18 .

\subsection{Synthesis of Carboxymethyl Chitosan-11-Mercapto Undecanoic Acid Conjugates (CMCS-MUA)}

CMCS-MUA was synthesized though the amidation reaction between the amino group of CMCS and the terminal carboxyl group of MUAaccording to the relevant literature information [21]. In brief, the obtained and dried CMCS $(0.1 \mathrm{~g})$ was dissolved in distilled water $(10 \mathrm{~mL})$ while being stirred for $24 \mathrm{~h}$. MUA dissolved in 5 $\mathrm{mL}$ of dimethylsulfoxide (DMSO) was treated with EDC. $\mathrm{HCl}$ and NHS for $45 \mathrm{~min}$ for the activation of carboxyl groups of MUA. Then the resulting MUA solution was added dropwise into the CMCS solution. The reaction mixture was continually stirred for $6 \mathrm{~h}$ at room temperature in the dark. Afterwards, the solution was dialyzed and lyophilized. The whole process was carried out under nitrogen protection to minimize oxidation of thiol groups. In the feed molar ratios of 1:2, 1:6, and 1:10 between carboxyl group of MUA and amino group of CMCS, three samples were synthesized and named as CMCS-MUA-1, CMCS-MUA-2 and CMCS-MUA-3, respectively. The amount of covalently attached 11-Mercapto undecanoic acid with free thiol groups on the conjugates was determined with Ellman's reagent (DTNB, 5, 5'-dithio-bis-(2-nitrobenzoic acid)) [22]. Their contents of free thiol groups ( $\mu$ mol SH/g polymer) were $137.819,107.334$, and 84.027 , respectively.

\subsection{Preparation of Disulfide Cross-Linked Nanoparticles}

$0.01 \mathrm{~g}$ of CMCS-MUA was dissolved in $10 \mathrm{~mL}$ deionized water under gentle shaking at $25^{\circ} \mathrm{C}$ in the absence of light for $24 \mathrm{~h}$, followed by ultrasonication using an ultrasonic cell crasher JY-92-2D at $40 \mathrm{~W}$ for 3 min to facilitate the oxidation reaction of thiol groups. To inhibit the heat buildup during sonication, the pulse function was used (pulse on $2.0 \mathrm{~s}$; pulse off $2.0 \mathrm{~s}$ ). The nanoparticles prepared using CMCS-MUA-1, CMCS-MUA-2 and CMCS-MUA-3 were named as NP1, NP2 and NP3, respectively.

\subsection{Characterization of Disulfide Cross-Linked Nanoparticles}

The zeta potential, mean size and size distribution of the nanoparticles were measured by DLS using a Zetasizer (Malvern ZS90). All measurements were done with a wavelength of $633.0 \mathrm{~nm}$ at $25^{\circ} \mathrm{C}$ with an angle detection of 
$90^{\circ}$, and each sample was repeatedly measured three times. Morphological evaluation of the nanoparticles was performed by TEM (TECNAI G220 S-TWIN, JEOL, Japan). For TEM analysis, sample solutions were dropped onto the carbon-coated 300 mesh copper grids and dried at $50^{\circ} \mathrm{C}$ and then examined without being stained.

\subsection{Stability Studies of Disulfide Cross-Linked Nanoparticles}

The stability of the disulfide cross-linked nanoparticles (NP1, NP2, NP3) was investigated by DLS. The nanoparticles were placed at room temperature for three months in buffer solution of $\mathrm{pH} 7.0$, and then the mean diameters, distributions and Zeta potentials were measured.

\section{7. pH-Sensitivity Studies of the Nanoparticles}

$0.01 \mathrm{~g}$ of lyophilized nanoparticles were dissolved in phosphate buffer solution (PBS) of five different $\mathrm{pH}$ values (4.0, 5.0, 6.0, 7.0, 8.0), followed by incubation in a shaking bed at $200 \mathrm{rpm}$ and $25^{\circ} \mathrm{C}$ for $24 \mathrm{~h}$. Then the mean diameters and size distributions of the nanoparticles were determined by Malvern ZS90.

\subsection{Glutathione-Responsiveness Studies of the Nanoparticles}

The study of glutathione-responsiveness of the nanoparticles was accomplished under the monitoring of DLS measurement. In brief, the lyophilized nanoparticles were dissolved in PBS of pH 7.0 and divided into three parts. One part without GSH was used as a control, and the other two parts were used adding GSH to reach the final concentrations of $10 \mu \mathrm{M}$ and $2 \mathrm{mM}$, respectively. Then the solutions were incubated in a shaking bed at $200 \mathrm{rpm}$ and $25^{\circ} \mathrm{C}$ for $24 \mathrm{~h}$. Afterwards the mean diameters and size distributions of the nanoparticles were also determined by Malvern ZS90.

\subsection{Preparation of Diuron-Loaded Nanoparticles}

Diuron, a hydrophobic compound, was employed as a model herbicide to investigate the drug loading and controlled release behavior. Typically, $0.1 \mathrm{~g}$ of CMCS-MUA and $10 \mathrm{mg}$ of diuron were dispersed in $100 \mathrm{~mL}$ deionized water under stirring for $24 \mathrm{~h}$ at room temperature in the absence of light, followed by ultrasonication at 40 $\mathrm{W}$ for $3 \mathrm{~min}$ to obtain the diuron-loaded nanoparticles dispersion solution. Insoluble, free diuron was removed by centrifugation of the dispersion solution at $2000 \mathrm{rpm}$ for $5 \mathrm{~min}$. Diuron-loaded nanoparticles were separated from the aqueous solution by centrifugation at 10,000 rpm for $15 \mathrm{~min}$, and washed with deionized water for three times to remove the diuron absorbed on the surface, and freeze-dried for $48 \mathrm{~h}$ in vacuum. The diuron-loaded nanoparticles prepared from NP1, NP2 and NP3 were named as DNP1, DNP2 and DNP3, respectively.

Diuron concentration in the supernatant and three washing solutions was determined by high-performance liquid chromatography (HPLC) using a UV detector. Using an acetonitrile-water mix (volume ratio 60:40) as the mobile phase at a flow rate of $1.0 \mathrm{~mL} / \mathrm{min}$, and a C18-bonded phase column, column temperature being $30^{\circ} \mathrm{C}$ and injection volume being $20 \mu \mathrm{L}$, diuron was analyzed on a Agilent 1100 HPLC-System at $250 \mathrm{~nm}$, its wavelength of maximum absorption. All measurements were performed in triplicate. Diuron quantification employed a validated calibration curve describing the relationships between the diuron concentration and the HPLC detector responses.

\subsection{Determination of Diuron-Loading Content and Encapsulation Efficiency}

Efficiency of the encapsulation process was characterized by two parameters: encapsulation efficiency $(E E)$ and loading efficiency $(L E)$.

$E E$ was defined as a ratio between the weight of diuron encapsulated and its total weight at the beginning of the process.

$$
E E=(\text { weight of encapsulated diuron/initial total weight of diuron }) \times 100 \%
$$

Weight of encapsulated diuron was calculated by the initial total amount of diuron subtracting the insoluble, free diuron and the residual diuron in the supernatant and three washing solutions.

$L E$ was defined as the amount of diuron encapsulated divided by the final weight of the dried diuron-loaded nanoparticles: 


$$
L E=(\text { weight of encapsulated diuron/weight of the dried nanoparticles }) \times 100 \%
$$

\subsection{In Vitro Release Study}

The study of in vitro release behavior of DNP1 was carried out in three different conditions. Typically, the dried DNP1 (20 mg) were dispersed in $5 \mathrm{~mL}$ of deionized water and placed in a dialysis membrane bag with a molecular cut-off of $5 \mathrm{kDa}$, tied and immersed into $50 \mathrm{~mL}$ of $\mathrm{pH} 7.0 \mathrm{PBS}$ media (simulating the cytoplasmic $\mathrm{pH}$ in plants cells [23])with different GSH concentrations: without GSH (as a control), $10 \mu \mathrm{M}$ GSH, $2 \mathrm{mM}$ GSH (simulating the GSH concentrations in various plant tissues), and shaken in a thermostatic rotary shaker at $25^{\circ} \mathrm{C}$ at a shaking speed of $25 \mathrm{rpm}$. At predetermined intervals, $5 \mathrm{~mL}$ of the release medium was removed and $5 \mathrm{~mL}$ of fresh medium was added into the system to maintain sink conditions. The amount of diuron in the release medium samples was determined by the HPLC method described above. All measurements were performed in triplicate.

In vitro release behavior of DNP1, DNP2 and DNP3 in the same media (pH 7.0, PBS, 2 mM GSH) was also investigated according to the method above, and free diuron as a control was also used for this study.

The cumulative drug release was calculated as follows:

Cumulative drug release

$=($ the amount of diuron released at the time $t /$ the initial amount of diuron in nanoparticles $) \times 100 \%$

\subsection{Herbicidal Activity Assays}

Herbicidal activity was evaluated by means of pre-emergence treatment [24] [25]. Pots $9.3 \mathrm{~cm}$ high, with an upper diameter of $12.5 \mathrm{~cm}$ and a lower diameter of $9.3 \mathrm{~cm}$, were filled with $600 \mathrm{~g}$ of plant substrate (Orgam Biomix). Fifteen seeds of the target species (Echinochloa crusgalli) and ten seeds of the nontarget species (Zea mays) were sown in each pot.

Two days after sowing, diuron and DNP1 were applied to the substrate by spraying using a concentration of the active principle equivalent to $3 \mathrm{~kg} / \mathrm{ha}$ (a typical concentration used in the field). NP1 was also applied, as well as water alone. After 10 days, the plants were collected, washed, dried, and weighted. Each test was performed in triplicate, and the results were expressed in terms of the mean and standard deviation of the dry mass. The statistical analysis employed analysis of variance (ANOVA) with the Tukey-Kramer post-test. These analyses were performed using GraphPad InStat v.3 software.

\section{Results and Discussion}

\subsection{Synthesis and Characterization of CMCS and CMCS-MUA}

CMCS and CMCS-MUA were synthesized according to the procedures schematically depicted in Figure 1. Firstly, as shown in Figure 1(a), CMCS was prepared by the modification of chitosan using chloroacetic acid. Then, as showing in Figure 1(b), the amine groups of CMCS reacted with the carboxyl groups of MUA to form amide bonds, resulting in an amphiphilic carboxymethyl chitosan derivative bearing both carboxyl groups and undecane chains.

The structures of CMCS and CMCS-MUA were characterized by Fourier transformed infrared (FT-IR) spectroscopy and Proton nuclear magnetic resonance $\left({ }^{1} \mathrm{H}\right.$ NMR) spectroscopy. The FT-IR spectra of CMCS (see curve a) and CMCS-MUA (see curve b) are shown in Figure 2. For curve a in Figure 2, two absorption bands at $1417 \mathrm{~cm}^{-1}$ and $1600 \mathrm{~cm}^{-1}$ may be belonged to the asymmetric stretching vibration and symmetric stretching vibration of carbonyl respectively, which showed that CMCS was successfully prepared. Compared with curve a, a new characteristic band at $2854 \mathrm{~cm}^{-1}$ was observed in curve b, which was assigned to the asymmetric stretching vibration of C-S bond in $\mathrm{CH}_{2}-\mathrm{SH}$. In addition, the intensity of the absorption band of $-\mathrm{CH}_{2}-$ at $2914 \mathrm{~cm}^{-1}$ had increased significantly. These results indicated that MUA was successfully grafted to CMCS.

The chemical structures of CMCS and CMCS-MUA determined by ${ }^{1} \mathrm{H}$ NMR spectroscopy are shown in Figure 3. Compared with curve a, new signal peaks in curve b at $\delta 1.28(-\mathrm{SH})$ and $\delta 2.59\left(-\mathrm{CH}_{2}\right.$ of $\left.-\mathrm{CH}_{2}-\mathrm{SH}\right)$ were observed, which further confirmed the successful preparation of CMCS-MUA.

\subsection{Formation and Characterization of Nanoparticles}

The schematic representation of assembly process of CMCS-MUA in aqueous solution was shown in Figure 4. 
(a)

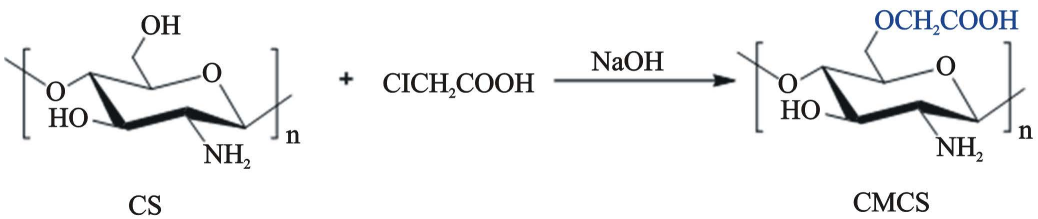

(b)

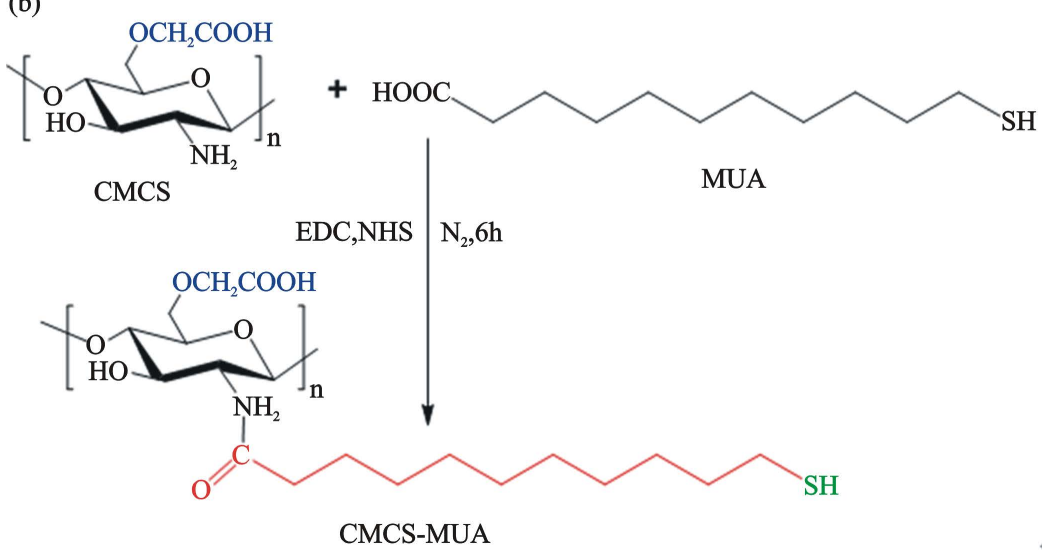

Figure 1. (a) Synthetic procedure of CMCS; (b) Synthetic procedure of CMCS-MUA.

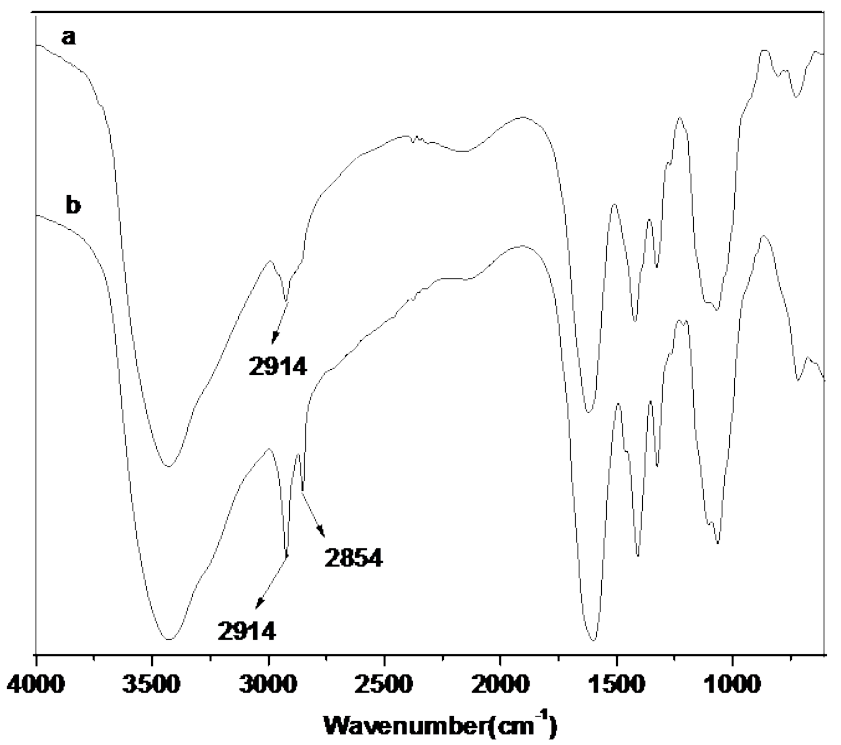

Figure 2. FT-IR spectra of: (a) CMCS; (b) CMCS-MUA.

A certain amount of CMCS-MUA were first dispersed in deionized water to self-assemble into nanoparticles with a core-shell structure, then the disulfide cross-linked core-shell nanoparticles were obtained by air-oxidation under ultrasonication. The TEM images of the resulting CMCS-MUA nanoaggregates were shown in Figure 5, which confirmed that the nanoaggregates had an approximate spherical geometry with obvious core-shell structure, and a relatively uniform size distribution of around $250 \mathrm{~nm}$ in diameter. The amount of remaining thiol groups in the cross-linked core-shell nanoparticles determined by Ellman's method is less than 12\%, which indicated that most of the thiols were oxidized to form disulfide bonds [26].

The prepared CMCS-MUA contains carboxyl groups and undecane chains which contribute to its amphiphilicity. Upon dispersing in aqueous solution, the undecane chains on CMCS-MUA tend to self-aggregate to form hydrophobic cores driven by hydrophobic interaction, and the hydrophilic segments with carboxyl groups tend 


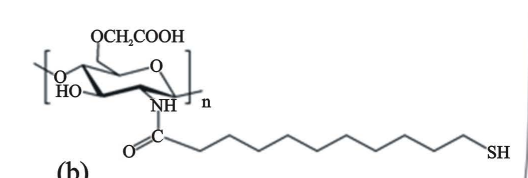

(b)
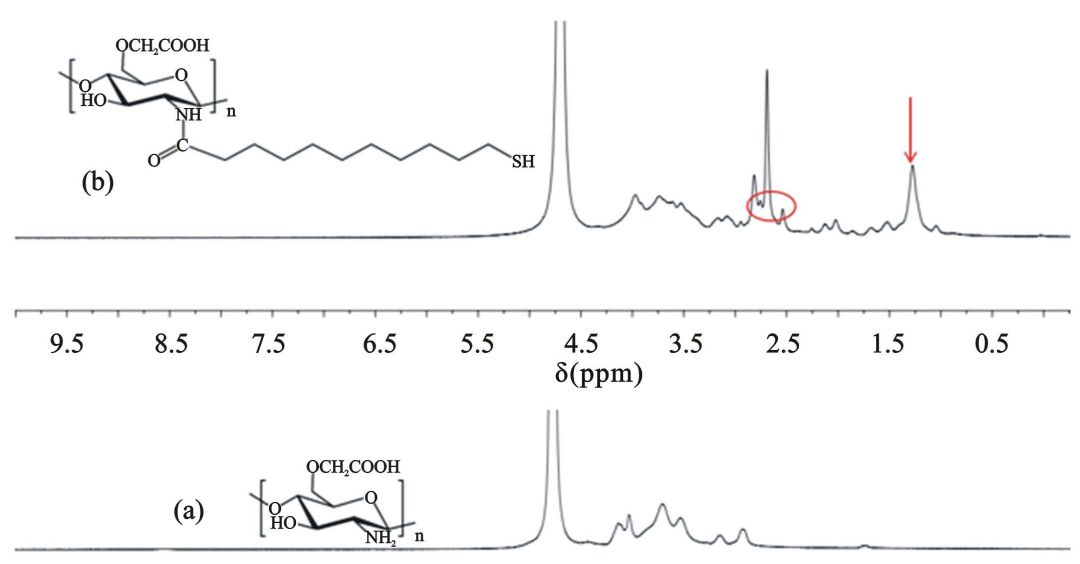

$\begin{array}{llllllllll}9.5 & 8.5 & 7.5 & 6.5 & 5.5 & \begin{array}{c}4.5 \\ \delta(\mathrm{ppm})\end{array} & 3.5 & 2.5 & 1.5 & 0.5\end{array}$

Figure 3. ${ }^{1} \mathrm{H}$ NMR spectra of: (a) CMCS; (b) CMCS-MUA.

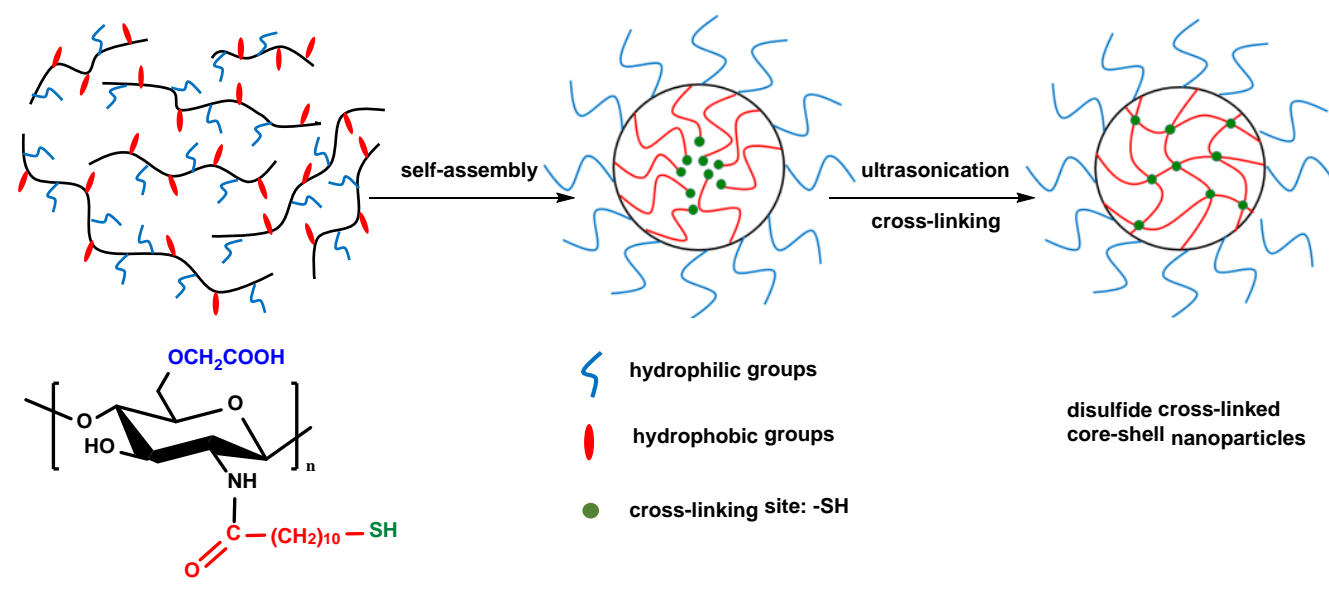

Figure 4. Assembly process of CMCS-MUA conjugates.

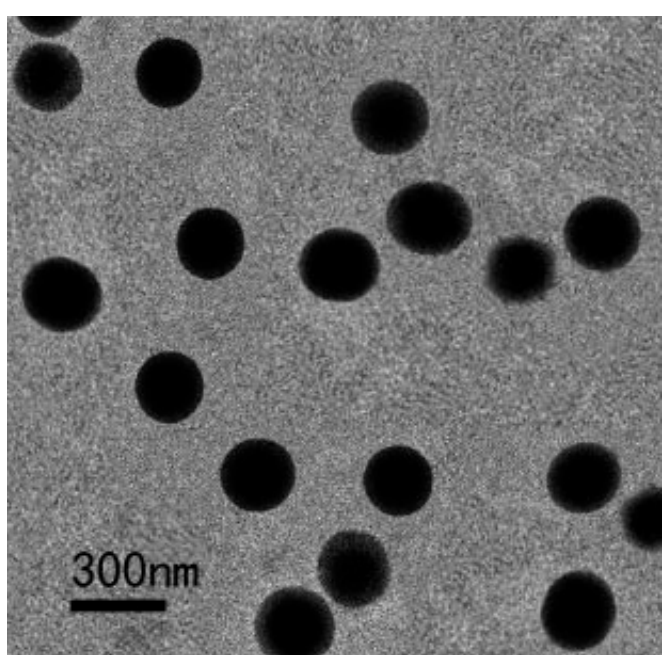

Figure 5. TEM images of self-aggregates prepared from CMCS-MUA conjugates. 
to stretch to form shells outside these hydrophobic cores, thus the core-shell nanostructure is obtained [27]. Then, under the treatment of ultrasonication, the neighbouring thiols in the hydrophobic cores are oxidized to form disulfide cross-linking bonds, resulting in a compact structure.

\subsection{Stability of the Disulfide Cross-Linked Nanoparticles}

Table 1 shows the mean diameters (MD) and distributions of the disulfide cross-linked nanoparticles before and after being placed for three months. It can be directly seen that the nanoparticles had little changes on sizes and polydispersity index (PDI) values, which suggested that the disulfide cross-linked nanoparticles had high stability. This may be attributed to the formation of intermolecular and intramolecular disulfide bonds. This kind of stability is of great significance for avoiding herbicides release before reaching the target sites. The Zeta potentials of nanoparticles are also shown in Table 1. It demonstrated that the nanoparticles had negative surface charges. In view of the Zeta potential theory, particles with Zeta potentials more positive than $+30 \mathrm{mV}$ or more negative than $-30 \mathrm{mV}$ are normally considered stable, so the nanoparticles prepared in this study have further proven to be quite stable.

\section{4. pH-Sensitivity of the Nanoparticles}

The pH-sensitivity of the disulfide cross-linked core-shell nanoparticles was studied by monitoring the mean nanoparticle size in the $\mathrm{pH}$ range of 4.0 to 8.0, in which precipitation was not observed. As shown in Figure 6, the diameters of the three samples increased with the increase of $\mathrm{pH}$ in the range of $\mathrm{pH} 4.0-7.0$ until reaching a maximum value at $\mathrm{pH}$ 7.0. This change could be ascribed to the following reason. In the range of $\mathrm{pH} 4.0-7.0$, the ionization degree of carboxyl groups on CMCS chains increases with the increase of $\mathrm{pH}$, which reduces the hydrogen bonds formed between carboxyl groups and increases electrostatic repulsion between ionized carboxyl groups. However when $\mathrm{pH}$ increased from 7.0 to 8.0, decreases in nanoparticle diameters were observed, this result might be attributed to the charge screening effect of the counter ions [28] [29].

Table 1. The mean diameters (MD), distributions and zeta potentials of nanoparticles at $\mathrm{pH} 7.0$ for their stability.

\begin{tabular}{ccccccc}
\hline \multirow{2}{*}{ Nanoparticles } & \multicolumn{3}{c}{ Before storage } & \multicolumn{3}{c}{ After storage (three months) } \\
\cline { 2 - 6 } & MD (nm) & PDI & Zeta potential (mV) & MD (nm) & PDI & Zeta potential (mV) \\
\hline NP1 & $225 \pm 1.9$ & $0.131 \pm 0.012$ & $-30.2 \pm 0.86$ & $228 \pm 1.5$ & $0.129 \pm 0.015$ & $-32.8 \pm 0.43$ \\
NP2 & $319 \pm 1.3$ & $0.092 \pm 0.006$ & $-33.1 \pm 1.10$ & $322 \pm 1.4$ & $0.106 \pm 0.008$ & $-31.6 \pm 0.26$ \\
NP3 & $490 \pm 0.9$ & $0.023 \pm 0.009$ & $-30.6 \pm 0.63$ & $496 \pm 1.1$ & $0.028 \pm 0.009$ & $-31.2 \pm 0.18$ \\
\hline
\end{tabular}

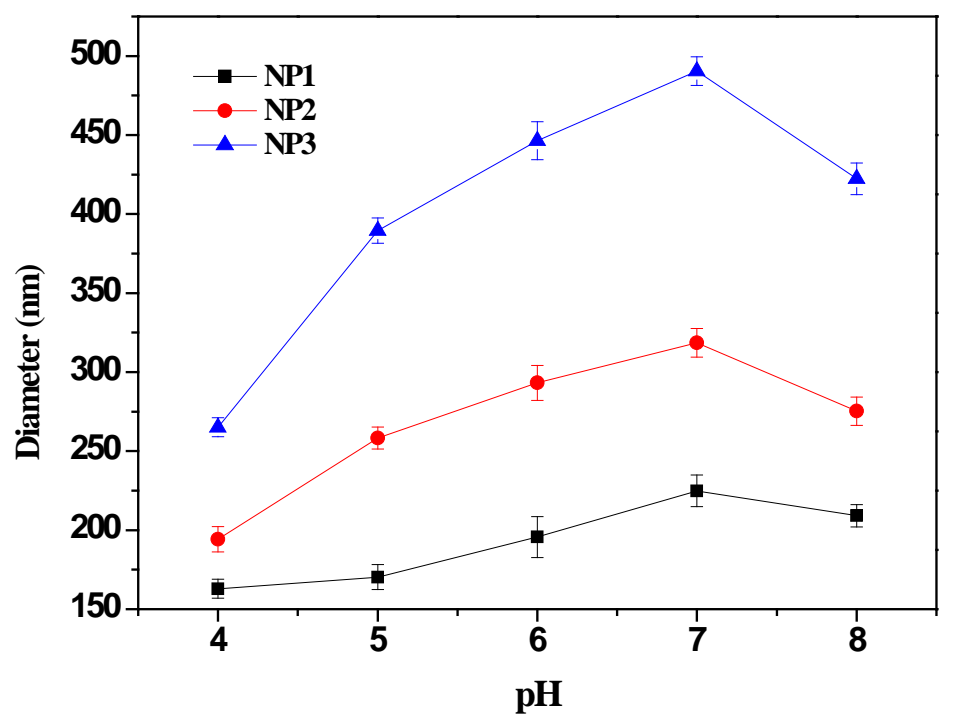

Figure 6. pH-sensitivity of the disulfide cross-linked core-shell nanoparticles. 
Additionally, it was also discovered that with the $\mathrm{pH}$ value increasing from 4.0 to 7.0 , the diameters of three samples (NP1, NP2 and NP3) increased by 1.38, 1.64 and 1.85 times, respectively, and the extent of the change was decreased in the following order: NP3 $>$ NP2 $>$ NP1. This result may be ascribed to the cross-linking degree. The more MUA substitution the sample had, the more disulfide cross-linking bonds it could form, which resulted in a more compact structure, so the $\mathrm{pH}$ sensitivity decreased with the increase of the cross-linking degree.

\subsection{Glutathione-Responsiveness of the Nanoparticles}

The mean diameters and distributions of nanoparticles at $\mathrm{pH}$ 7.0 PBS containing GSH of different concentrations $(0,10 \mu \mathrm{M}, 2 \mathrm{mM})$ for $24 \mathrm{~h}$ are shown in Table 2. For each of the three samples, the mean diameter and PDI value at $10 \mu \mathrm{M} \mathrm{GSH}$ almost keep unchanged compared to that of the control group without GSH, while an obvious increase at $2 \mathrm{mM} \mathrm{GSH}$ was observed. This result illustrates that the nanoparticles can remain relatively stable in pH 7.0 PBS without GSH or with a relatively low concentration of GSH $(10 \mu \mathrm{M})$, while at a relatively high concentration of GSH (2 mM), the disulfide cross-linking bonds can be cleaved, resulting in an increase in mean size and PDI.

\subsection{Diuron Loading and In Vitro Release}

To investigate the drug-loading capacity of the nanoparticles, diuron, a hydrophobic compound, was employed as a model herbicide to load into the nanoparticles in suit. Table 3 showed the diuron loading efficiency (LE) and encapsulation efficiency (EE) of nanoparticles as well as the solubility of diuron. As can be seen from Table 3, NP3 expressed a lower encapsulation efficiency of around $19.87 \%$. When the mole ratio of MUA substitution to the CMCS unit increased from 1:10 to 1:2, the encapsulation efficiency of diuron increased from $19.87 \%$ to $78.28 \%$, and the diuron loading efficiency increased from $1.95 \%$ to $7.26 \%$. This result may be attributed to the increase of the hydrophobic volumes of inner cores [30] [31]. In addition, compared with the solubility of free diuron in water ( $4.2 \mathrm{mg} / 100 \mathrm{~g} \mathrm{H}_{2} \mathrm{O}$ ), a significantly improvement was observed for NP1 up to $11.8 \mathrm{mg} / 100 \mathrm{~g}$ $\mathrm{H}_{2} \mathrm{O}$.

In order to further investigate the in vitro release behavior in different concentrations of GSH, DNP1 was chosen as the object of study. As it had been reported that the cytoplasmic $\mathrm{pH}$ in plants was maintained at relatively constant values close to neutrality despite the existence of pH-perturbing processes [23], and GSH was present at concentration of $2 \mathrm{mM}$ to $3 \mathrm{mM}$ in various plant tissues [15]. Based on this, the $\mathrm{pH}$ 7.0 PBS containing different concentrations of GSH $(0,10 \mu \mathrm{M}, 2 \mathrm{mM})$ were chosen to simulate the environment in plant cells. Figure 7 revealed the diuron release from DNP1 in pH 7.0 PBS with GSH of different concentrations. As can be seen, the percentage of the cumulative release at $2 \mathrm{mM} \mathrm{GSH}$ arrived at $58.18 \%$ after $24 \mathrm{~h}$, which was much higher than that of the control group without GSH (only 13.02\%), and the percentage of the cumulative release at $10 \mu \mathrm{M}$ GSH only arrived at $18.92 \%$, which was a little over the control group.

Table 2. The mean diameters and distributions of nanoparticles at pH 7.0 PBS containing different concentrations of GSH for $24 \mathrm{~h}$.

\begin{tabular}{ccccccc}
\hline \multirow{2}{*}{ Nanoparticles } & \multicolumn{2}{c}{$\mathrm{GSH}(0 \mu \mathrm{M})$} & \multicolumn{2}{c}{$\mathrm{GSH}(10 \mu \mathrm{M})$} & \multicolumn{2}{c}{$\mathrm{GSH}(2 \mathrm{mM})$} \\
\cline { 2 - 7 } & MD $(\mathrm{nm})$ & PDI & MD $(\mathrm{nm})$ & PDI & MD $(\mathrm{nm})$ & PDI \\
\hline NP1 & $225 \pm 1.9$ & $0.131 \pm 0.012$ & $235 \pm 1.8$ & $0.162 \pm 0.018$ & $298 \pm 2.1$ & $0.235 \pm 0.023$ \\
NP2 & $319 \pm 1.3$ & $0.092 \pm 0.006$ & $329 \pm 0.8$ & $0.123 \pm 0.006$ & $405 \pm 1.5$ & $0.199 \pm 0.016$ \\
NP3 & $490 \pm 0.9$ & $0.023 \pm 0.009$ & $503 \pm 1.2$ & $0.026 \pm 0.002$ & $586 \pm 1.3$ & $0.160 \pm 0.009$ \\
\hline
\end{tabular}

Table 3. The diuron loading efficiency and encapsulation efficiency of nanoparticles and solubility of diuron.

\begin{tabular}{ccccc}
\hline \multirow{2}{*}{ Nanoparticles } & MUA/CMCS unit & Loading efficiency & Encapsulation efficiency & $\begin{array}{c}\text { Solubility } \\
\left.\text { (diuronmg/100g } \mathrm{H}_{2} \mathrm{O}\right)\end{array}$ \\
\hline NP1 & $(\mathrm{mol} / \mathrm{mol})$ & $\%$ & $78.28 \pm 2.36$ & $11.828 \pm 1.654$ \\
NP2 & $1: 2$ & $7.26 \pm 1.53$ & $49.79 \pm 1.06$ & $8.979 \pm 0.872$ \\
NP3 & $1: 6$ & $4.74 \pm 1.08$ & $19.87 \pm 1.13$ & $5.987 \pm 0.256$ \\
\hline
\end{tabular}


The release mechanism should be explained from the following aspects. When in pH 7.0 PBS without GSH, the release of diuron might be attributed to the diffusion of diuron on the surface of the nanoparticles out of the dialysis membrane. When the concentration of GSH increased to a high level namely $2 \mathrm{mM}$, the disulfide bonds were cleaved to some degree, which destroyed the disulfide cross-linking network structure and therefore made it easier for diuron to release outward [32]-[34]. These results indicated that these disulfide cross-linked nanoparticles can be used as carriers for herbicides in agriculture by taking advantage of the GSH concentration in plant cells.

Figure 8 demonstrated the release profiles from DNP1, DNP2, and DNP3 in pH 7.0 PBS with $2 \mathrm{mM} \mathrm{GSH}$. It was interesting to note an initial fast release of diuron during the first 4 hours, followed by a more gradual and sustained release phase for the following $19 \mathrm{~h}$. For DNP1, about $42 \%$ of diuron still remained in the nanoparticles even after $24 \mathrm{~h}$. The initial fast release of diuron might be due to the diffusion of diuron on the surface of the nanoparticles out of the dialysis membrane. This was also consistent with the rate of diffusion across the dialysis membrane for free diuron. Then the following relatively slow release could be dominated by the degree of hydrophobic MUA substitution. As can be seen, the cumulative rates of diuron over time from diuron-loaded nanoparticles in $\mathrm{pH} 7.0$ PBS with $2 \mathrm{mM}$ GSH were ranked in the following order: DNP3 > DNP2 > DNP1, which might be ascribed to the increase of the disulfide cross-linking degree. The nanoparticle with a higher

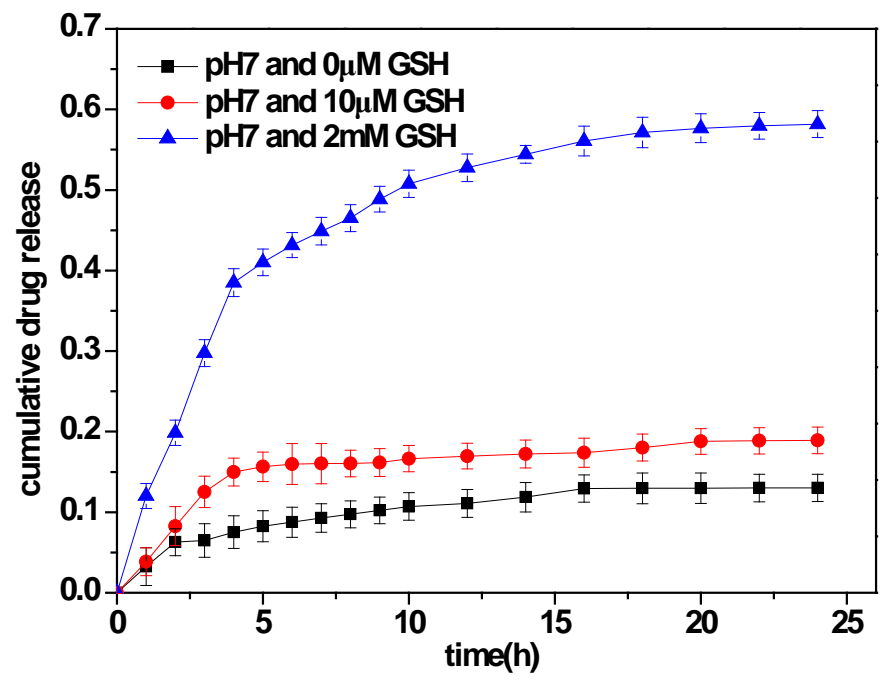

Figure 7. Diuron release profiles from DNP1 in pH 7.0 PBS with GSH of different concentrations. $\bullet \mathrm{pH} 7$ and $0 \mu \mathrm{M}$ GSH; $\bullet \mathrm{pH} 7$ and $10 \mu \mathrm{M} \mathrm{GSH} ; \boldsymbol{\Delta} \mathrm{pH} 7$ and $2 \mathrm{mM} \mathrm{GSH}$.

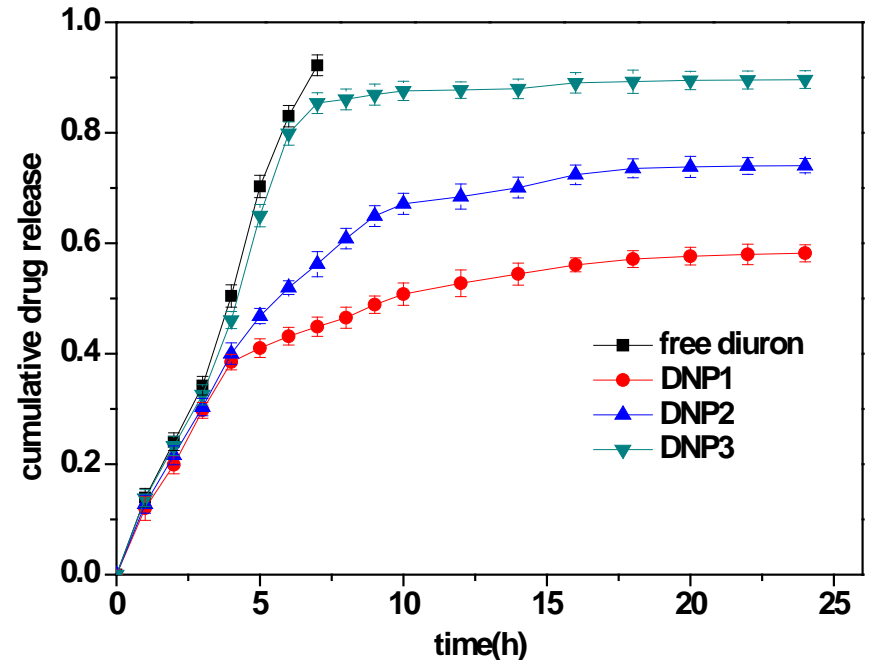

Figure 8. Diuron release profiles from diuron-loaded nanoparticles in pH 7.0 PBS with 2 mM GSH. free diuron; $\bullet$ DNP1; $\Delta$ DNP2; $\nabla$ DNP3 
degree of disulfide cross-linking exhibited a smaller particle size and a more compact network structure, which would make it more difficult for diuron to release.

\subsection{Evalution of Herbicidal Activity}

The herbicidal activity of DNP1, NP1, as well as free diuron, were investigated using two species, one a target species (Echinochloa crusgalli), and the other a nontarget species (Zea mays). The application concentration of diuron (equivalent to $3.0 \mathrm{~kg} / \mathrm{ha}$ ) was the same as that employed commercially in field crops.

Diuron can be used to control weeds in maize plantations by pre-emergence treatment. Maize is resistant to diuron because of the presence in this species of an enzyme, cytochrome P450 monooxygenase, which is able to degrade diuron [35] [36]. The effects on the two species are illustrated in Figure 9 (Figure 9(a) and Figure 9(b)), where it can be seen that there were no obvious changes in the dry weight of Zea mays, while in the case of Echinochloa crusgalli, there was a greater effect for DNP1, compared to diuron alone. For the target species (Echinochloa crusgalli), as shown in Figure 9(b), all the formulations showed effect, relative to the control, with the exception of the NP1. So it is speculated that the nanoparticles prepared in this paper showed no phytotoxic effect in Echinochloa crusgalli. The greater effectiveness of DNP1 against the target plant could be explained by the following reasons: the presence of the nanoparticles acted to increase the interaction with the plant root, or that the nanoparticles were absorbed by the roots, hence improving delivery of the diuron to the plant tissues [37], and once the diuron-loaded nanoparticles arrived at the plant tissues, they were exposed to an environment of 2 - $3 \mathrm{mM} \mathrm{GSH}$, at which disulfide cross-linking bonds could be cleaved to some degree, and thus diuron was released in a controlled manner. Another important point is that encapsulation is likely to have protected diuron against physico-chemical and micro-biological degradation.

\section{Conclusions}

In our work, amphipathic CMCS-MUA bearing both hydrophilic carboxymethyl groups and hydrophobic MUA substitutions was successfully synthesized. A nanosized glutathione-responsive controlled release system for diuron was also successfully fabricated by self-assembling in aqueous solutions. The results of TEM proved that the self-assembled nanoparticles exhibited spherical morphology with a core-shell configuration. The ionizable carboxy groups furnished the nanoparticles with $\mathrm{pH}$-sensitivity, and the reducible disulfide bonds provided nanoparticles with glutathione -responsiveness. The in vitro release profiles of DNP1 in pH 7.0 PBS with GSH of different concentrations $(0,10 \mu \mathrm{M}, 2 \mathrm{mM})$ revealed that the release rate of diuron strongly depended on the GSH concentration, and a relatively high concentration of GSH namely $2 \mathrm{mM}$ can trigger the gradual and sustained release of diuron from nanoparticles. The results of in vitro release for DNP1, DNP2, and DNP3 in pH 7.0 PBS with $2 \mathrm{mM}$ GSH demonstrated that the release rate of diuron from nanoparticles over time decreased with the increase of the degree of hydrophobic MUA substitution. Herbicidal activity assays performed with pre-emergence treatment of target species (Echinochloa crusgalli) showed the effectiveness of diuron-loaded nanoparticles. Assays with non-target species (Zea mays) showed that the diuron-loaded nanoparticles did not affect plant growth.

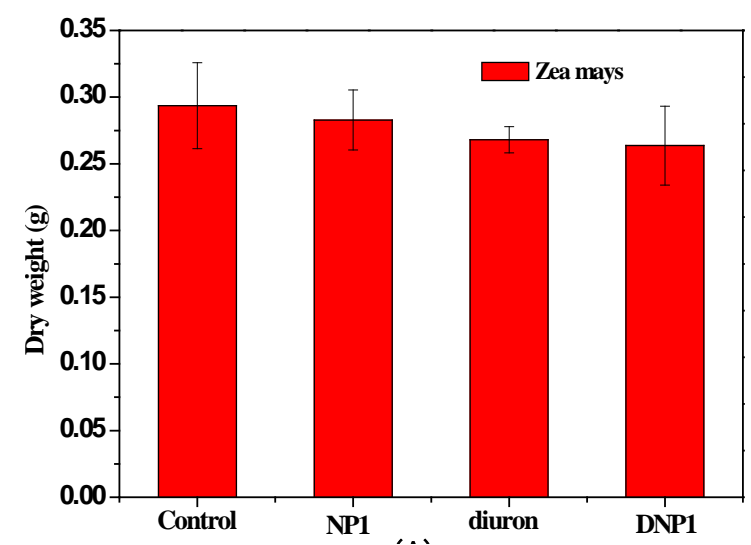

(a)

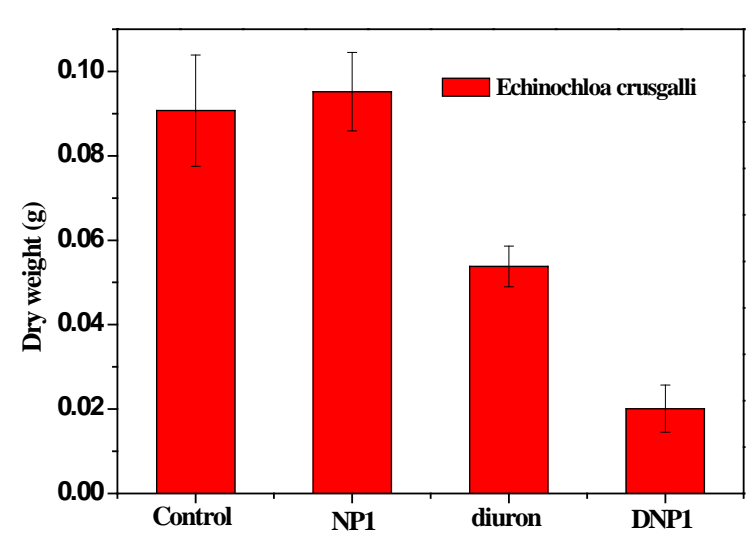

(b)

Figure 9. Dry weight after pre-emergence treatment of Zea mays (a) and Echinochloa crusgalli (b). 
In a word, disulfide cross-linked diuron-loaded nanoparticles prepared in this study can release diuron at 2 mM GSH, and almost no release of diuron at the environment without GSH or with a relatively low concentration of GSH such as $10 \mu \mathrm{M}$. So nanoparticles fabricated in this study could have great potential for controlled release of herbicides. Before getting to plant cells, herbicides could not leak out, and once entering plant cells, they are exposed to an environment of $2-3 \mathrm{mM} \mathrm{GSH}$, at which disulfide cross-linking bonds could be cleaved to some degree, and thus release herbicides in a controlled manner, so the disulfide cross-linked nanoparticles could be a potential candidate for controlled release of herbicides.

\section{Acknowledgements}

This work was financially supported by the Natural Science Foundation of China (No. 51373130 No. 51273156, and No. 51303145).

\section{References}

[1] Cotterill, J.V., Wilkins, R.M. and da Silva, F.T. (1996) Controlled Release of Diuron Granules Based on a Lignin Matrix System. Journal of Controlled Release, 40, 133-142. http://dx.doi.org/10.1016/0168-3659(95)00188-3

[2] Fernandez-Perez, M., Villafranca-Sanchez, M., Gonzalez-Pradas, E. and Flores-Cespedes, F. (1999) Controlled Release of Diuron from an Alginate-Bentonite Formulation: Water release Kinetics and Soil Mobility Study. Journal of Agricultural and Food Chemistry, 47, 791-798. http://dx.doi.org/10.1021/jf980878y

[3] Agnihotri, S.A. and Aminabhavi, T.M. (2004) Controlled Release of Clozapine through Chitosan Microparticles Prepared by a Novel Method. Journal of Controlled Release, 96, 245-259. http://dx.doi.org/10.1016/j.jconrel.2004.01.025

[4] Grillo, R., Pereira Anderson do Espirito, S., de Melo Nathalie Ferreira, S., Porto Raquel, M., Feitosa Leandro, O., Tonello Paulo, S., et al. (2011) Controlled Release System for Ametryn Using Polymer Microspheres: Preparation, Characterization and Release Kinetics in Water. Journal of Hazardous Materials, 186, 1645-1651. http://dx.doi.org/10.1016/j.jhazmat.2010.12.044

[5] Grillo, R., dos Santos Nathalia Zocal, P., Maruyama Cintia, R., Rosa Andre, H., de Lima, R. and Fraceto Leonardo, F. (2012) Poly(E-caprolactone) Nanocapsules as Carrier Systems for Herbicides: Physico-Chemical Characterization and Genotoxicity Evaluation. Journal of Hazardous Materials, 231-232, 1-9. http://dx.doi.org/10.1016/j.jhazmat.2012.06.019

[6] Roy, A., Singh, S.K., Bajpai, J. and Bajpai, A.K. (2014) Controlled Pesticide Release from Biodegradable Polymers. Central European Journal of Chemistry, 12, 453-469. http://dx.doi.org/10.2478/s11532-013-0405-2

[7] Riggle, B.D. and Penner, D. (1990) The Use of Controlled-Release Technology for Herbicides. Reviews of Weed Science, 5, 1-14.

[8] Yu-Ling, L., Li, Z., Zhaozhong, L., Ru, C., Fenghua, M., Jing-Hao, C., et al. (2009) Reversibly Stabilized Multifunctional Dextran Nanoparticles Efficiently Deliver Doxorubicin into the Nuclei of Cancer Cells. Angewandte Chemie, International Edition, 48, 9914-9918. http://dx.doi.org/10.1002/anie.200904260

[9] Tian-Bin, R., Yue, F., Zhong-Hai, Z., Lan, L. and Yong-Yong, L. (2011) Shell-Sheddable Micelles Based on StarShaped Poly(E-caprolactone)-SS-poly(ethyl glycol) Copolymer for Intracellular Drug Release. Soft Matter, 7, 23292331. http://dx.doi.org/10.1039/c1sm05020f

[10] Russo, A., DeGraff, W., Friedman, N. and Mitchell, J.B. (1986) Selective Modulation of Glutathione Levels in Human Normal versus Tumor Cells and Subsequent Differential Response to Chemotherapy Drugs. Cancer Research, 46, 2845-2848.

[11] Arrick, B.A. and Nathan, C.F. (1984) Glutathione Metabolism as a Determinant of Therapeutic Efficacy: A Review. Cancer Research, 44, 4224-4232.

[12] Schafer, F.Q. and Buettner, G.R. (2001) Redox Environment of the Cell as Viewed through the Redox State of the Glutathione Disulfide/Glutathione Couple. Free Radical Biology \& Medicine, 30, 1191-1212. http://dx.doi.org/10.1016/S0891-5849(01)00480-4

[13] Ojima, I. (2008) Guided Molecular Missiles for Tumor-Targeting Chemotherapy-Case Studies Using the SecondGeneration Taxoids as Warheads. Accounts of Chemical Research, 41, 108-119. http://dx.doi.org/10.1021/ar700093f

[14] Bauhuber, S., Hozsa, C., Breunig, M. and Goepferich, A. (2009) Delivery of Nucleic Acids via Disulfide-Based Carrier Systems. Advanced Materials, 21, 3286-3306. http://dx.doi.org/10.1002/adma.200802453

[15] Ball, L.A., Gian, P., Bechtold, U., Creissen, G., Funck, D., Jimenez, A., et al. (2004) Evidence for a Direct Link between Glutathione Biosynthesis and Stress Defense Gene Expression in Arabidopsis. The Plant Cell, 16, 2448-2462. 
http://dx.doi.org/10.1105/tpc.104.022608

[16] Yin, Y.H., Lv, X.L., Tu, H.W., Xu, S. and Zheng, H. (2010) Preparation and Swelling Kinetics of pH-Sensitive Photocrosslinked Hydrogel Based on Carboxymethyl Chitosan. Journal of Polymer Research, 17, 471-479. http://dx.doi.org/10.1007/s10965-009-9333-4

[17] Upadhyaya, L., Singh, J., Agarwal, V. and Tewari, R.P. (2013) Biomedical Applications of Carboxymethyl Chitosans. Carbohydrate Polymers, 91, 452-466. http://dx.doi.org/10.1016/j.carbpol.2012.07.076

[18] Liu, X.F., Guan, Y.L., Yang, D.Z., Li, Z. and Yao, K.D. (2001) Antibacterial Action of Chitosan and Carboxymethylated Chitosan. Journal of Applied Polymer Science, 79, 1324-1335. http://dx.doi.org/10.1002/1097-4628(20010214)79:7<1324::AID-APP210>3.0.CO;2-L

[19] Anitha, A., Divya Rani, V.V., Krishna, R., Sreeja, V., Selvamurugan, N., Nair, S.V., et al. (2009) Synthesis, Characterization, Cytotoxicity and Antibacterial Studies of Chitosan, O-Carboxymethyl and N,O-Carboxymethyl Chitosan Nanoparticles. Carbohydrate Polymers, 78, 672-677. http://dx.doi.org/10.1016/j.carbpol.2009.05.028

[20] Chen, X.G. and Park, H.J. (2003) Chemical Characteristics of O-Carboxymethyl Chitosans Related to the Preparation Conditions. Carbohydrate Polymers, 53, 355-359. http://dx.doi.org/10.1016/S0144-8617(03)00051-1

[21] Gao, C., Liu, T., Dang, Y.H., Yu, Z.Y., Wang, W., Guo, J.J., et al. (2014) pH/Redox Responsive Core Cross-Linked Nanoparticles from Thiolated Carboxymethyl Chitosan for in Vitro Release Study of Methotrexate. Carbohydrate Polymers, 111, 964-970. http://dx.doi.org/10.1016/j.carbpol.2014.05.012

[22] Perera, G., Barthelmes, J. and Bernkop-Schnuerch, A. (2010) Novel Pectin-4-Aminothiophenole Conjugate Microparticles for Colon-Specific Drug Delivery. Journal of Controlled Release, 145, 240-246. http://dx.doi.org/10.1016/j.jconrel.2010.04.024

[23] Kurkdjian, A. and Guern, J. (1989) Intracellular pH: Measurement and Importance in Cell Activity. Annual Review of Plant Physiology and Plant Molecular Biology, 40, 271-303. http://dx.doi.org/10.1146/annurev.pp.40.060189.001415

[24] Pereira, A.E.S., Grillo, R., Mello, N.F.S., Rosa, A.H. and Fraceto, L.F. (2014) Application of Poly(Epsilon-Caprolactone) Nanoparticles Containing Atrazine Herbicide as an Alternative Technique to Control Weeds and Reduce Damage to the Environment. Journal of Hazardous Materials, 268, 207-215. http://dx.doi.org/10.1016/j.jhazmat.2014.01.025

[25] Grillo, R., Pereira, A.E.S., Nishisaka, C.S., de Lima, R., Oehlke, K., Greiner, R., et al. (2014) Chitosan/Tripolyphosphate Nanoparticles Loaded with Paraquat Herbicide: An Environmentally Safer Alternative for Weed Control. Journal of Hazardous Materials, 278, 163-171. http://dx.doi.org/10.1016/j.jhazmat.2014.05.079

[26] Garcia, R., Jose L., Parra, A. and Aleman, J. (2008) Efficient Synthesis of Disulfides by Air Oxidation of Thiols under Sonication. Green Chemistry, 10, 706-711. http://dx.doi.org/10.1039/b800705e

[27] Chang, D., Lei, J., Cui, H.R., Lu, N., Sun, Y.J., Zhang, X.H., Gao, C., Zheng, H. and Yin, Y.H. (2012) Disulfide Cross-Linked Nanospheres from Sodium Alginate Derivative for Inflammatory Bowel Disease: Preparation, Characterization, and in Vitro Drug Release Behavior. Carbohydrate Polymers, 88, 663-669.

http://dx.doi.org/10.1016/j.carbpol.2012.01.020

[28] Hua, S.B. and Wang, A.Q. (2009) Synthesis, Characterization and Swelling Behaviors of Sodium Alginate-g-Poly (Acrylic Acid)/Sodium Humate Superabsorbent. Carbohydrate Polymers, 75, 79-84. http://dx.doi.org/10.1016/j.carbpol.2008.06.013

[29] Pourjavadi, A., Sadeghi, M. and Hosseinzadeh, H. (2004) Preparation, Swelling Behavior, Salt- and pH-Sensitivity of Partially Hydrolyzed Crosslinked Carrageenan-Graft-Polymethacrylamide Superabsorbent Hydrogel. Polymers for Advanced Technologies, 15, 645-653. http://dx.doi.org/10.1002/pat.524

[30] Liu, K.-H., Chen, B.-R., Chen, S.-Y. and Liu, D.-M. (2009) Self-Assembly Behavior and Doxorubicin-Loading Capacity of Acylated Carboxymethyl Chitosans. The Journal of Physical Chemistry B, 113, 11800-11807. http://dx.doi.org/10.1021/jp902103p

[31] Liu, T.-Y., Chen, S.-Y., Lin, Y.-L. and Liu, D.-M. (2006) Synthesis and Characterization of Amphiphatic Carboxymethyl-Hexanoyl Chitosan Hydrogel: Water-Retention Ability and Drug Encapsulation. Langmuir, 22, 9740-9745. http://dx.doi.org/10.1021/la061471n

[32] Cheng, R., Feng, F., Meng, F., Deng, C., Feijen, J. and Zhong, Z. (2014) Glutathione-Responsive Nano-Vehicles as a Promising Platform for Targeted Intracellular Drug and Gene Delivery. Journal of Controlled Release, 152, 2-12.

[33] Yang, D., Chen, W.L. and Hu, J.H. (2014) Design of Controlled Drug Delivery System Based on Disulfide Cleavage Trigger. The Journal of Physical Chemistry B, 118, 12311-12317. http://dx.doi.org/10.1021/jp507763a

[34] Meng, F.H., Hennink, W.E. and Zhong, Z.Y. (2009) Reduction-Sensitive Polymers and Bioconjugates for Biomedical Applications. Biomaterials, 30, 2180-2198. http://dx.doi.org/10.1016/j.biomaterials.2009.01.026

[35] Barrett, M. (1995) Metabolism of Herbicides by Cytochrome P450 in Corn. Drug Metabolism and Drug Interactions, 12, 299-315. http://dx.doi.org/10.1515/dmdi.1995.12.3-4.299 
[36] Barrett, M. (1997) Herbicide Selectivity Mechanisms in Maize: Using What We Know for the Future. Brighton Crop Protection Conference-Weeds, 2, 587-596.

[37] de Oliveira, J.L., Vangelie Ramos Campos, E., da Silva, C.M.G., Pasquoto, T., Lima, R. and Fraceto, L.F. (2015) Solid Lipid Nanoparticles Co-Loaded with Simazine and Atrazine: Preparation, Characterization, and Evaluation of Herbicidal Activity. Journal of Agricultural and Food Chemistry, 63, 422-432. http://dx.doi.org/10.1021/jf5059045 